\title{
Fabrication and Test of an Optical Magnetic Mirror
}

\author{
John G. Hagopian, Patrick A. Roman, Shahram Shiri, Edward J. Wollack, \\ NASA Goddard Space Flight Ctr. (Greenbelt, MD USA); \\ Madhumita Roy, \\ U.S. Army Research Lab. (Adelphi, MD USA)
}

1. INTRODUCTION

Traditional mirrors at optical wavelengths use thin metalized or dielectric layers of uniform thickness to approximate a perfect electric field boundary condition. The electron gas in such a mirror configuration oscillates in response to the incident photons and subsequently re-emit fields where the propagation and electric field vectors have been inverted and the phase of the incident magnetic field is preserved. We proposed fabrication of sub-wavelength-scale conductive structures that could be used to interact with light at a nano-scale and enable synthesis of the desired perfect magneticfield boundary condition. In a magnetic mirror, the interaction of light with the nanowires, dielectric layer and ground plate, inverts the magnetic field vector resulting in a 0 degree phase shift upon reflection. Geometries such as split ring resonators and sinusoidal conductive strips were shown to demonstrate magnetic mirror behavior in the microwave [1] and then in the visible [2]. Work to design, fabricate and test a magnetic mirror began in 2007 at the NASA Goddard Space Flight Center (GSFC) under an Internal Research and Development (IRAD) award. Our initial nanowire geometry was sinusoidal but orthogonally asymmetric in spatial frequency, which allowed clear indications of its behavior by polarization. We report on the fabrication steps and testing of magnetic mirrors using a phase shifting interferometer and the first far-field imaging of an optical magnetic mirror.

\subsection{Description of interaction of the magnetic field of light with periodic metallic nanostructures}

Periodic metallic nanostructures can interact with the magnetic field of light in ways that can produce effects not found in ordinary matter. The development of these meta-materials provides opportunities to create novel optical detectors, solar cells and imaging devices. In the limit where the period of the surface structures are smaller than the wavelength, diffraction does not occur and an effective medium model can describe the structure. If the structure is lossless and its surface impedance is large compared to free space the incident wave will be reflected without a phase change. A perfect electric conductor (PEC) reflects electromagnetic waves without loss and changes the sign of the electric field (i.e., reflection coefficient $R=-1$ and phase change of $180 \mathrm{deg})$. A surface, which reflects a wave without a sign change, $(R=1$, zero phase change) is known as a perfect magnetic conductor (PMC). A trivial PMC can be realized with a quarter wavelength delay in front of a ground plane. This simple magnetic mirror configuration is commonly used as an element of a matching network in far-IR absorber structures for bolometric sensors. At microwave frequencies, compact-planar alternatives based upon frequency-selective surfaces (FSS) above a conducting ground plane have been proposed and investigated (e.g., [3-5] and others). The concept of suppressing surface waves (plasmons) using corrugated metallic structures characterized by a high surface impedance which electromagnetically serves as an artificial magnetic conductor over a finite bandwidth [6-8] has also been historically employed. The resulting band-gap structures have been used for antenna miniaturization, surface wave suppression, and diffraction reduction. From a circuit theory perspective, all of these structures are electromagnetic variations on a similar theme.

The initial design concepts at GSFC of our proposed magnetic mirror, operating at visible wavelengths, and using nanorings, were modified due to the difficulty in fabricating millions of nano-rings in favor of fabricating a continuous sine wave pattern, similar to the "fish scale" nanostructures fabricated by other researchers. The nanostructure will not diffract if the freespace wavelength of the incident wave is greater than twice the period of the magnetic mirror's effective unit cell. It is important to note that the size of the unit cell is a function of the dielectric index and the geometry of the pattern employed - this is not to say that a device will not work above this condition but, that its response can be shown to be multi-moded and that diffractive losses may occur in this limit. The object of the IRAD was to determine if the classical diffraction of such a device in the far-field was different than that of an ordinary mirror and to design various nanowire patterns and evaluate them for diffraction suppression. 


\section{NANOSTRUCTURE MIRROR DESIGN AND FABRICATION}

There were several constraints that drove the design of our initial magnetic mirror. Since proof of magnetic mirror behavior required verification of a 0 degree phase shift upon reflection, we needed to characterize the phase shift of reflection relative to that of an ordinary mirror. Therefore, the mirror was designed to operate at $633 \mathrm{~nm}$ the wavelength of our phase shifting interferometer. By designing the resonant frequency of the device to be different in orthogonal directions, we could verify performance by comparing the phase shift of the orthogonal polarizations. In addition, we wanted a device that would be compatible with an optical imaging configuration, to enable inspection of the far-field diffraction pattern. The far-field diffraction pattern of an optical system is dominated by the characteristics of the limiting aperture of the system. Since fabrication of a magnetic mirror at visible wavelengths requires e-beam writing, the device figure had to be flat and its size limited. Precision writing of an e-beam pattern to the edge of the device could be difficult, therefore the design required that the magnetic mirror had to be fabricated with a black surround that did not reflect light. The diffraction from an ordinary mirror results in a pattern that is comprised of a core image and alternating dark and bright diffraction orders. For a circular aperture, the image is called the Airy pattern and comprised of a core surrounded by dark and light rings of decreasing intensity. The use of a square aperture shape concentrates the diffracted light into brighter orders that are easier to image; therefore we selected a square mirror configuration. The device also had to be within the capability of GSFC to perform the majority of the fabrication operations for budgetary considerations. It was determined that we could perform all required fabrication steps except the electron-beam, (ebeam), writing needed to pattern the nanowires. We contracted with the Army Research Laboratory (ARL), which was local to GSFC to perform the e-beam writing. E-beam writing is an expensive operation, so our proof of concept devices had a $3 \mathrm{~mm}$ square active area with the black surround making the full device $1 \mathrm{~cm}$ square.

\subsection{Magnetic mirror design and fabrication}

Our first magnetic mirror design utilized a unit cell that was asymmetric in spatial frequency in orthogonal directions, with its resonant frequency designed to reflect light of $633 \mathrm{~nm}$ wavelength in one polarization and $1.0 \mu \mathrm{m}$ wavelength in the other. Figure 1 is a depiction of unit cell design as modeled in COMSOL, a multi-physics modeling package with some nanostructure modeling capabilities. The initial design utilized a pseudo-sinusoidal pattern of nanowires approximately $80 \mathrm{~nm}$ in width and height. Due to the requirement to have a black surround for imaging tests, we had to create a black surround on the devices after nano-wire fabrication. In 2007 our IRAD team discovered that we could tune mulitwalled carbon nanotube geometry during growth to

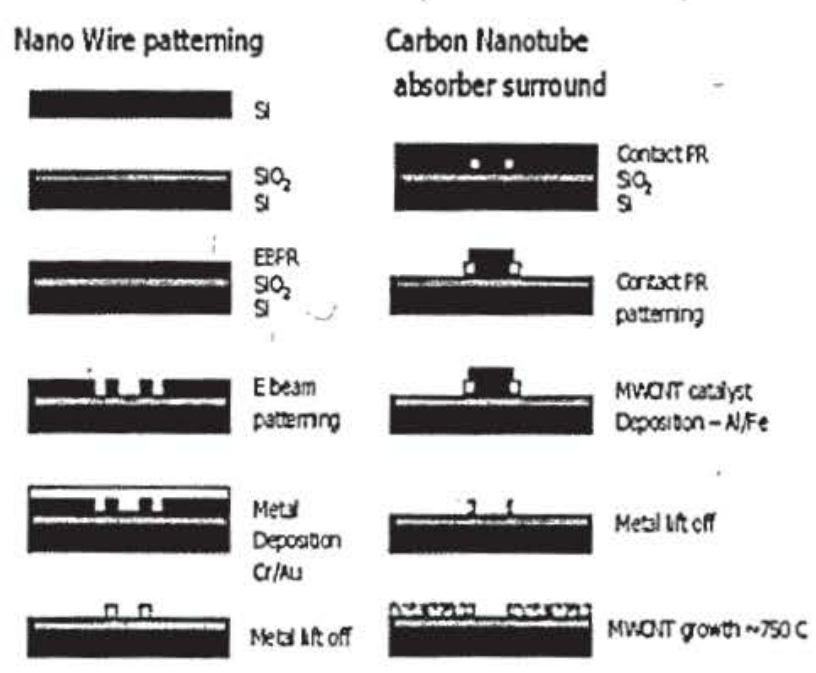

Figure 2. Device Fabrication Steps
30 Model of Nanomes Cell, Electric Field Discribution

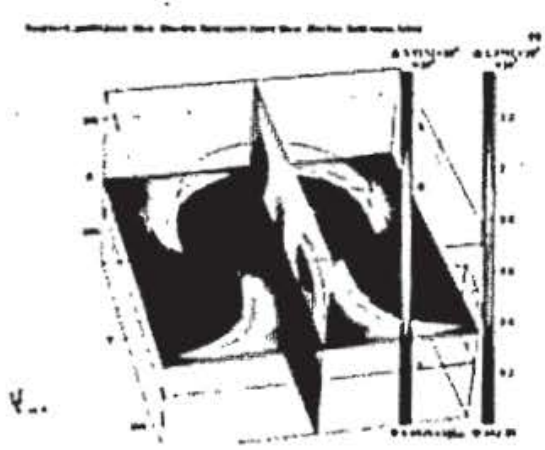

Figure 1. Nanowire Unit Cell Geometrv

exceptionally black nanotube films to create this black surround. (We subsequently modified this process for use with other substrates and to qualify it for stray light control on space flight instrumentation [9].) Since the growth of carbon nanotubes requires temperatures of $750^{\circ}$ Centigrade, our pathfinder device utilized gold nanowires instead of aluminum (aluminum is preferred because it has more free electrons per volume). Unfortunately, we found that nanoscale gold "wetted" or nearly melted even at reduced growth temperatures of $650^{\circ} \mathrm{C}$, well below its bulk melting point of $1064^{\circ} \mathrm{C}$. This is one of the peculiar behaviors of materials at nanoscales that must be taken into consideration during device design. 
Therefore our first operational device utilized aluminum nanowires and an alternate black surround- Acktar litho black. The steps required for device fabrication are shown in Figure 2. Silicon wafers were screened interferometrically to select those that were the flattest to ensure near diffraction limited performance during image testing. Aluminum was then evaporatively deposited on the wafer to serve as a ground plate. A dielectric layer was then deposited on the aluminized substrate; several processes were used over the course of our investigation, including spin-on-glass and evaporatively deposited magnesium fluoride or silicon oxide. The wafers were then delivered to ARL along with our coded nano pattern. ARL performed e-beam writing of the pattern along with reference fiducials on the wafers. The wafers were then metalized at GSFC and a lift-off process was performed to produce the nanowire structures; a scanning electron microscope (SEM) image of the lift-off process and nanowires are shown in Figure 3. The metalized fiducials were used as alignment references to register a lithographic mask to the e-beam written wafer, treated with photoresist. This process allowed the surround region to be blackened using the process of our choice and patterning of 4 devices per wafer: 2 nanostructured mirrors and 2 control mirrors. The wafer was then diced into the 4 devices and the protective layer removed. This process allows fabrication of magnetic mirror devices and control mirrors with identical clear-aperture size, shape, black surround, base metal and dielectric-layer thickness and composition.

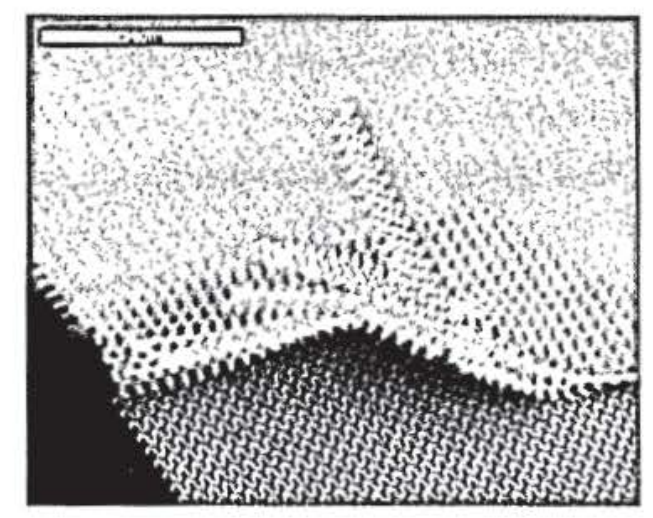

\subsection{Magnetic mirror performance verification}

The initial plan to verify magnetic mirror behavior was to simultaneously measure the phase shift of the control device relative to the magnetic mirror device using a simultaneous phase-shifting Fizeau interferometer. The ordinary mirror should create a 180 degree phase shift of the wavefront, while the magnetic mirror produces a 0 degree phase shift. Alignment of the base of the mirrors to a backing reference mirror would allow a common reference surface for comparison. It was determined that this provision was not required in our first mirror because of a deficiency in the Acktar litho black surround.

While the lithographic Acktar litho black, did appear quite black, when it was applied to the polished silicon wafer, the resulting smooth dielectric surface reflected quite well. The interferometer was able to acquire fringes of both the surround and clear apertures of both the magnetic mirror device. The interferometer produces a surface map of the device under test and can remove residual aberrations to
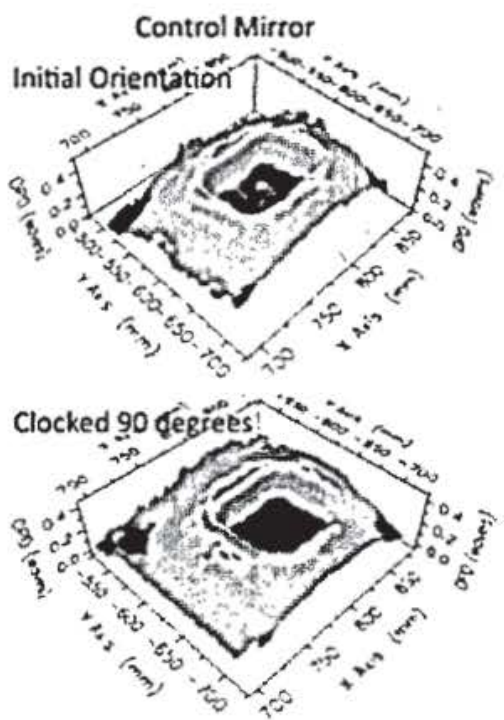

Figure 4. Surface Map of Control

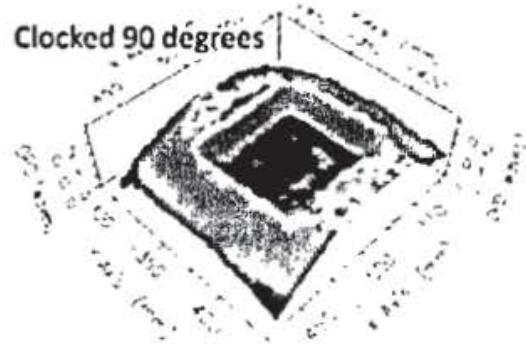

Figure 5. Surface Map of Magnetic Mirror Device \#2 allow better visualization of the surface. Figure 4 shows the surface map of the control mirror, in 2 orientations corresponding to the two polarization axes of the device. The surface map shows a depression where the $3 \mathrm{~mm}$ clear aperture is located and a higher region corresponding to the surround. Since the interferometer operates at a single wavelength, it is not possible to determine the absolute height difference between the clear aperture corresponding to the ordinary mirror and the surround. Upon rotation, the mirror surface map simply rotates as expected, demonstrating identical behavior with orthogonal polarizations. 
The behavior of the magnetic mirror, our Device $\# 2$ can be seen in Figure 5 . In the polarization corresponding to the resonant frequency of the sinusoidal nanopattern, the clear aperture appears as an elevated region relative to the surround, while the orthogonal orientation produces a depressed region similar to that seen in the control mirror. This indicates fundamentally different behavior in the device-resonant axis, relative to the non-resonant axis. In the resonant axis, the magnetic mirror region differs by 180 degrees of phase with the respect to both the orthogonal axis and the control mirror, indicating a 0 degree phase shift of the incident wavefront. It is important to note that identical processing of the control mirror removes variables such as dielectric birefringence and thickness variations.

\section{SECOND-GENERATION MAGNETIC MIRROR DESIGN AND IMAGING TESTS}

Several design changes were implemented to allow far-field image testing of the next generation magnetic mirror. These modifications were implemented to allow the device to be resonant in both polarizations for interferometric testing and to create a black surround for image testing. In addition, refinements were made to the diffraction evaluation test bed to allow testing at other wavelengths and to provide adequate dynamic range to evaluate the diffraction pattern to several diffraction orders. Although, the core image contains $80 \%$ of the energy, higher orders are attentuated rapidly, with $4 \%$, $1.5 \%, .8 \%$ of the energy in first 3 diffraction maximia.

\subsection{Device modifications and interferometric testing}

The second-generation device was created with a smaller unit cell, with resonant frequencies corresponding to a wavelengths below $633 \mathrm{~nm}$. While magnetic mirror behavior in both polarizations cannot be identical due to the geometry of a sinusoidal pattern, the spacing of the sine wave patterns determines the resonant frequency in the orthogonal direction. The unit cell dimension was decreased to $440 \mathrm{~nm}$, therefore the e-beam resolution was adjusted to create a $40 \mathrm{~nm}$ nanowire width. Unfortunately, this results in write times that were 4 times greater for creating same $3 \mathrm{~mm}$-square active area. A second refinement was to follow the deposition of the base aluminum layer on the wafer directly with a silicon-oxide dielectric deposition to simplify the fabrication process. The final modification of the process to make our "diffraction optimized" device was to create a nanoetch black surround to replace the Acktar litho black for imaging purposes. An image of the device submerged in solvent under high-intensity illumination is shown in Figure 6; in this image only a portion of the surround is shown. The Moiré pattern is due to aliasing of the detector and the image color is false because of the device being submerged.

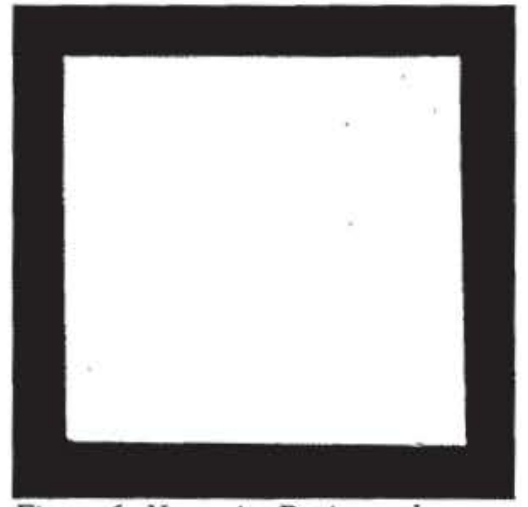

Figure 6. Nanowire Device and

The addition of the nano-etch surround and the resonant frequency polarization symmetry does not allow direct verification of the magnetic mirror behavior, as performed in Device \#2. Prior to fabrication of the full-frame high-resolution device, a subaperture Pathfinder was fabricated with a reflective surround, to allow verification of magnetic mirror behavior.

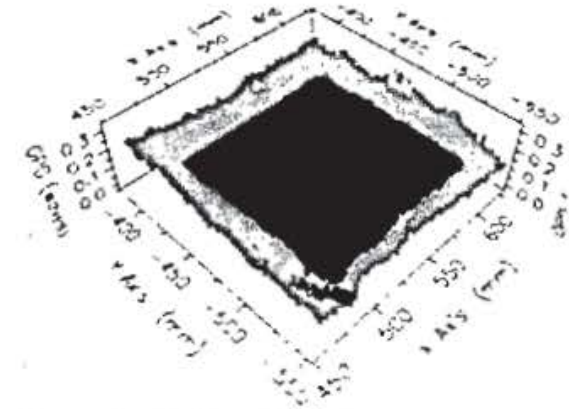

Figure 8. Surface Map of Device \#4

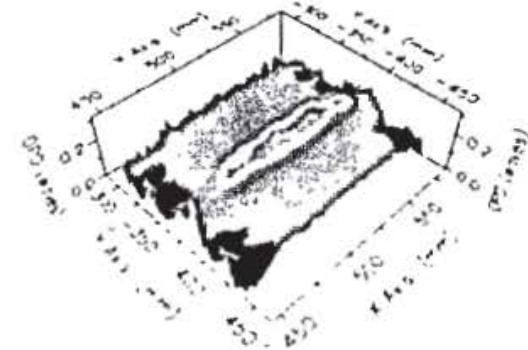

Figure 7. Surface Map of Pathfinder \#4 and on a more distorted wafer, it is difficult to assess the amount of phase change of the magnetic mirror relative to the surround, but adequate to continue with the full device. Device \#4, was successfully fabricated, diced and placed in the interferometer test configuration to verify magnetic mirror behavior. Figure 8 shows a interferometrically obtained surface plot of Device \#4 that does not change with orientation, demonstrating equivalent performance in both polarizations. Since there is no 
specular reflection from the nano-etch surround, the surround area that appears as a raised frame is not real data and does not provide a relevant phase reference.

\subsection{Imaging test bed}

A test bed was built to allow evaluation of the imaging characteristics of the nanostructure mirror devices and controls. A schematic of the optical configuration and photograph is shown in Figure 9. The test bed source consists of a spatially filtered point source utilizing a tunable laser. In a spatial filter, a laser source is focused by a microscope objective onto a pinhole matched to the magnification of the objective. The focus is adjusted to the pinhole, to reject higher-order spatial frequency content to create a near ideal spherical wavefront. A parabolic mirror was then aligned so that its focus was at the position of the pinhole, to create a collimated beam or plane wave. The plane wave propagates back towards the point source and is reflected off of the device under test, which is off the central axis of the system and tilted to direct the beam to a focus mirror and then off of a fold mirror to a CCD camera. The device under test is the limiting aperture of the system, at least 25 times smaller than the other optics in collimated space.

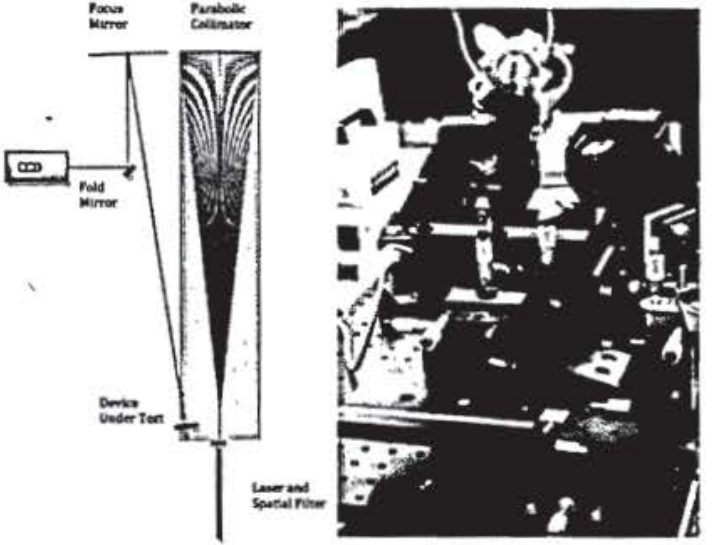

Figure 9. Diffraction Imaging Test Bed The system was aligned using standard metrology processes and verified using the Fizeau interferometer and was determined to have less than $1 / 40$ of a wave of aberration over the test aperture. The CCD camera is a cooled device with 14 bits of dynamic range or 16384 gray levels, sufficient to discern the $10^{\text {th }}$ diffraction order maxima of the square device in our test bed, which is $1 / 1000$ as bright as the peak. The polarization of the collimated beam is controlled using an input polarizer before the spatial filter, with a polarizer also placed after the device under test, also in collimated space. A single-wavelength tooling laser, with extreme wavelength and pointing stability was used in intial tests. Device \#4 was also tested with a tunable laser to investigate the imaging characteristics of the device at shorter than the design wavelength of $633 \mathrm{~nm}$.

\subsection{Device \#4 imaging characteristics}

Device \#4 was carefully aligned to the test bed and the CCD adjusted to focus the image. The input and output polarization was varied and in-focus images collected. Tests were intially performed at the design wavelength of $633 \mathrm{~nm}$, with the intensity of the laser attenuated using a neutral-density filter prior to the spatial filter. The attenuation and CCD exposure was set so that the maximum pixel was near full well, to maximize the dynamic range of the measurement. Images consisted of 128 averaged frames, and background

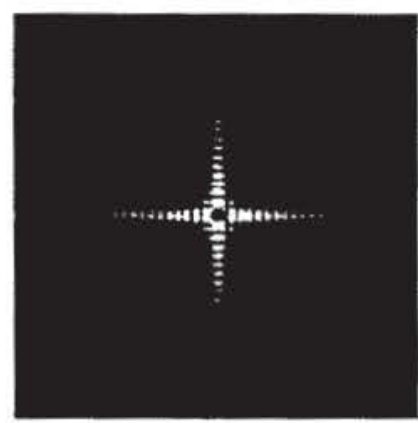

Figure 11. Log Ideal Mirror images with the laser blocked were also collected with the same number of averaged frames, for background subtraction to optimize signal to noise. After a complete data set of Device \#4 was acquired, the control device was

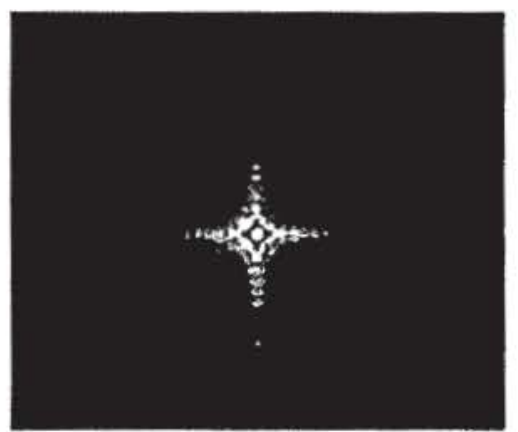

Figure 10. Log Stretch Image Device \#4 aligned to the system and a data set was acquired using the same technique. Frames were processed using National Institute of Health's (NIH) Image J software with the image calculator function to subtract the background. The resulting images were upconverted to 16 bits and had low background noise. A log stretch of the resulting images was performed using the ImageJ math function. A log stretched, background subtracted 16 color image produced by the system with Device \#4 installed appears in Figure 10. It clearly shows the central core and diffracted orders of the system 
and magnetic mirror device. An $\mathrm{x}$-axis slice of the image was acquired using the line tool in ImageJ and the plot profile used to acquire the log-stretched pixel value of the profile for comparison between images and ideal log stretched

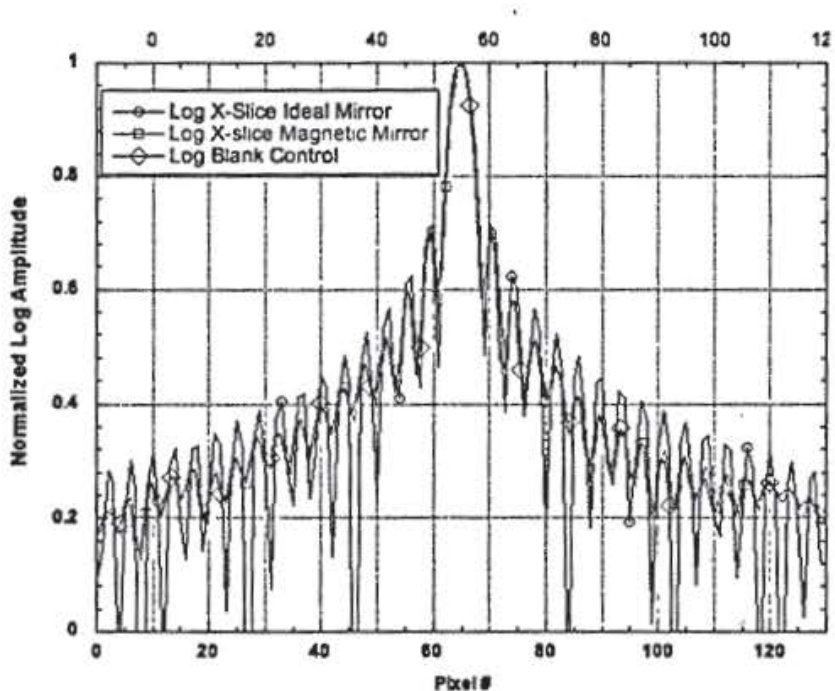

Figure 12. X-Slice of Ideal, Magnetic Mirror and Control Mirror images. Figure 11 is a log-stretch image of a modeled ideal mirror in the test bed configuration. The same process was used to generate an $\mathrm{x}$-slice of the control device. Optical Research Associates Code V software was used to generate an $\mathrm{x}$-slice of an ideal mirror in the test bed. The ideal image $x-$ slice was processed using the ImageJ algorithm for direct comparison with the log stretch $\mathrm{x}$-slices the magnetic mirror and the control device. The results are shown in Figure 12; it is interesting that the magnetic mirror and control mirror display near identical diffraction across many orders, but that both devices are lower than the theoretical limit beyond the first diffraction order. This result is probably due

to a non-linearity in the $\mathrm{CCD}$ or some slight variation in the processing of the $\mathrm{x}$-slice data. This discrepancy illustrates the value of having a control mirror processed identically to the test mirror for characterization This indicates that for our magnetic mirror, the far-field diffraction is identical as that for a electric field mirror. Additional images were obtained at other wavelengths by adjusting the tunable laser and realigning the spatial filter. It is interesting that images taken at $596 \mathrm{~nm}$ wavelength displayed higher-order diffraction in both $\mathrm{x}$ and $\mathrm{y}$ axes indicating that the device elements have an effective cell larger than that wavelength. Since the device is tilted away from normal incidence by about 7 degrees, the effective size changes, but diffraction can be seen in both the off axis and on axis directions. Figure 13 is a log stretch background subtracted image taken at $596 \mathrm{~nm}$ showing the diffraction pattern presumably from the nanowire pattern.

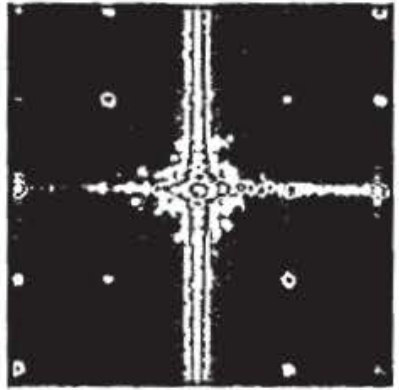

Figure 13. Magnetic Mirror Log Image at $596 \mathrm{~nm}$ Additional images were obtained at $650 \mathrm{~nm}$ using the tunable laser source at 635 and 643 $\mathrm{nm}$ and no higher order diffraction was observed.

\section{CONCLUSIONS}

Over the course of three years we were able to design, fabricate and test a number of nanostructured devices. Magnetic mirror performance at $633 \mathrm{~nm}$ was observed in Device \#2 in one orientation (or polarization) despite the fact that the unit cell was significantly larger than the design wavelength. This validates that magnetic mirror behavior may be more a function of nanowire and dielectric geometry than just unit cell size. Device \#4 was optimized to allow magnetic mirror performance at the design wavelength of $633 \mathrm{~nm}$, but during Pathfinder testing appeared to be less efficient than Device \#2. Therefore, further testing and refinements to the COMSOL model which is just reaching a level of maturity, needs to be performed to determine the cause of these departures from expectations. In imaging tests, the far-field diffraction pattern of Device \#4 showed essentially no difference from the control device, indicating that no significant departure from theory (for an E-field mirror) was present. Future efforts to create different imaging devices will focus on various changes in geometry of the nano patterns; the tools we have demonstrated in the fabrication and test of our devices are well suited to these investigations. 


\section{ACKNOWLEDGEMENTS}

The authors would like to recognize Peter Hughes the NASA GSFC Chief Technologist and his staff Deborah Amato and Barbie Medina for continued support through the GSFC IRAD program. The authors also would like to recognize David Aronstein for providing Code V modeling of and ideal mirror in the diffraction evaluation test bed.

\section{REFERENCES}

[1] V. A. Fedotov, A. V. Rogacheva, N. I. Zheludeva, P. L. Mladyonov and S. L. Prosvirnin, "Mirror that does not change the phase of reflected waves", APPLIED PHYSICS LETTERS 88, 0911192006.

[2] A. S. Schwanecke, V. A. Fedotov, V.V. Khardikov, S.L. Prosvirnin, Y. Chen and N.I. Zheludev, "Optical magnetic mirrors", J. Opt. A: Pure Appl. Opt. 9 (2007) L1-L2; doi:10.1088/1464-4258/9/1/L01

[3] A. Monorchio, G. Manava, G., and L.Lanuzza, 'Synthesis of artificial magnetic conductors by using multilayered frequency selective surfaces', IEEE Antennas Wirel. Propag. Lett., 2002, 1, pp. 196-199

[4] A.P. Feresidis, G. Goussetis, S. Wang, and J.C. Vardaxoglu, 'Artificial magnetic conductor surfaces and their application to low-profile high-gain planar antennas', IEEE Trans. Antennas Propag., 2005, 53, (1), pp. 209-215

[5] D.J. Kern, D.H Werner, A. Monorchio, L. Lanuzza, and M.J. Wilhelm, 'The design synthesis of multiband artificial magnetic conductors using high impedance frequency selective surfaces', IEEE Trans. Antennas Propag., $2005,53,(1)$, pp. 8-17

[6] W. Rotman, "A study of single-surface corrugated guides," Proc. IRE, vol. 39, pp. 952-959, Aug. 1951.

[7] R. Elliot, "On the theory of corrugated plane surfaces," IRE Trans. Antennas Propagat., vol. 2, pp. 71-81, Apr. 1954.

[8] S. Lee and W. Jones, "Surface waves on two-dimensional corrugated surfaces," Radio Sci., vol. 6, pp. 811-818, 1971.

[9] J.G.Hagopian, S.A.Getty, M.Quijada, J Tveekrem, R. Shiri, P.A. Roman, J. Butler; G. Georgiev; J. Livas; C. Hunt; A. Maldonado; S. Talapatra; X. Zhang; S. J. Papadakis; A. H. Monica; D. Deglau"Multiwalled carbon nanotubes for stray light suppression in space flight instruments "Proc. SPIE 7761, 77610F (San Diego, 2010) 